\title{
Numerical research of the gas hydrate dissociation to gas and Ice in a reservoir during the gas extraction
}

\author{
Nail Musakaev ${ }^{1,2 *}$, and Stanislav Borodin ${ }^{1}$ \\ ${ }^{1}$ Tyumen Branch of Khristianovich Institute of Theoretical and Applied Mechanics of SB RAS, \\ 625026 Tyumen, Russia \\ ${ }^{2}$ Industrial University of Tyumen, 625000 Tyumen, Russia
}

\begin{abstract}
The peculiarities of the gas hydrate decomposition to gas and ice during the gas extraction from the porous medium initially saturated with methane and its hydrate are studied. It is shown that at a negative initial reservoir temperature, the gas hydrate decomposition always occurs with the formation of ice either on the frontal surface or in the extended region. It is established that the extended region of hydrate dissociation is characteristic for large values of the mass flow rate of the gas extraction.
\end{abstract}

\section{Introduction}

At present, hydrocarbon gas is one of the most important sources for the chemical and energy industries. At the same time, the largest reserves of methane on our planet are in the form of gas hydrates [1]. For the extraction of natural gas from the gas hydrate deposit it is necessary to decompose the hydrate directly in the reservoir and this can be achieved by the thermal action on the hydrate-saturated reservoir and/or by lowering the pressure at the producing well bottom. If the hydrate decomposition produces gas and ice rather than gas and water, then this allows reducing the energy costs for the gas hydrate deposits development, since the specific heat of the phase transition "hydrate - ice and gas" is significantly lower than that of "hydrate - water and gas" [2]. It is worth noting that the extraction of hydrocarbon raw stock from the gas hydrate deposits is a very complex technological problem that needs thorough and comprehensive theoretical research [1].

The research of the gas hydrate decomposition process in porous structures is presented in [2-5]. In these papers the mathematical models are proposed, and the analytical solutions in plane-parallel and axisymmetric formulations are constructed. It is shown that the gas hydrate decomposition can occurs either at the frontal surface or in the extended region. In this paper the mathematical model of the gas hydrate decomposition in the case of the negative initial reservoir temperature (below $0^{\circ} \mathrm{C}$ ) is constructed. This model takes into account the main features of the process, namely nonisothermal filtering of gas, phase transitions, real gas properties, adiabatic cooling effect, and Joule-Thomson effect.

*Corresponding author: musakaev@ikz.ru 


\section{Mathematical model}

Let the horizontal porous reservoir is initially saturated with methane and its hydrate, whose pressure $p_{0}$ and temperature $T_{0}$ correspond to the thermodynamic conditions of their existence in the free state:

$$
t=0, r_{w}<r<R_{k}: T=T_{0}<0{ }^{\circ} \mathrm{C}, p=p_{0}, S_{h}=S_{h 0}, S_{g}=1-S_{h 0},
$$

where $t$ is the time; $r$ is the radial coordinate; $r_{w}$ and $R_{k}$ are the well and reservoir radiuses; $S_{j}(j=i, h, g)$ is the $j$-th phase saturation in pores ( $i$ - ice, $h$-hydrate, $g-$ gas); $S_{h 0}$ is the initial hydrate saturation. The coordinate $r$ is measured from the central well axis.

The gas production occurs with a constant mass flow rate $Q$ (per unit of the well height), and there is no heat flow at the well boundary:

$$
t>0, r=r_{w}: \quad r \frac{\partial p^{2}}{\partial r}=\frac{Q z \mu_{g} R_{g} T}{\pi k S_{g}^{3}}, \frac{\partial T}{\partial r}=0 .
$$

Here $k$ is the absolute reservoir permeability; $\mu_{g}$ and $z$ are the dynamic viscosity of gas and compressibility factor; $R_{g}$ is the specific gas constant.

The gas hydrate decomposes due to the lowering of the pressure below the equilibrium pressure, and three characteristic zones can appear in a reservoir (Fig. 1). More specifically, the dissociation of the gas hydrate begins at the boundary between the second and third zones $\left(r=r_{(d)}\right)$ and ends at the boundary between the first and second zones $\left(r=r_{(n)}\right)$. The hydrate saturation in the third zone at all points is constant and equal to initial value $\left(S_{h 0}\right)$.

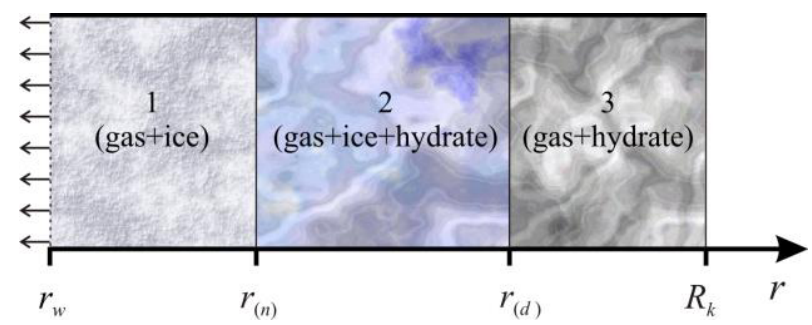

Fig. 1. Scheme of the gas hydrate dissociation into gas and ice occurring in a reservoir.

The conditions on the right boundary for temperature and pressure:

$$
t>0, r=R_{k}: \frac{\partial p}{\partial r}=0, \quad \frac{\partial T}{\partial r}=0 .
$$

The following assumptions are accepted: the porosity of a reservoir $m$ is constant; the skeleton of a porous medium, gas hydrate and ice are incompressible and immovable; the temperatures of the porous medium and its saturants are equal (one-temperature model).

The system of basic equations describing the processes of filtration and heat transfer during the gas hydrate decomposition in a porous medium is based on the laws of conservation of mass and energy, the Darcy law and the equation of the gas state $[2,6,7]$ :

$$
\frac{\partial}{\partial t}\left(\rho_{g} S_{g}+\rho_{h} S_{h} G\right)+\frac{1}{r} \frac{\partial}{\partial r}\left(r \rho_{g} S_{g} v_{g}\right)=0
$$




$$
\begin{gathered}
\frac{\partial}{\partial t}\left(\rho_{i} S_{i}+\rho_{h} S_{h}(1-G)\right)=0, \\
m S_{g} v_{g}=-\frac{k_{g}}{\mu_{g}} \frac{\partial p}{\partial r}, \\
\rho c \frac{\partial T}{\partial t}=\frac{1}{r} \frac{\partial}{\partial r}\left(r \lambda \frac{\partial T}{\partial r}\right)+\frac{\partial}{\partial t}\left(m \rho_{h} L_{h} S_{h}\right)-\rho_{g} c_{g} m S_{g} v_{g}\left(\frac{\partial T}{\partial r}+\varepsilon \frac{\partial p}{\partial r}\right)+\rho_{g} c_{g} m S_{g} \eta \frac{\partial p}{\partial t}, \\
p=z \rho_{g} R_{g} T, \quad k_{g}=k S_{g}^{3}, \quad S_{g}+S_{i}+S_{h}=1, \\
\varepsilon=-\frac{1}{\rho_{g} c_{g}} \frac{T}{z}\left(\frac{\partial z}{\partial T}\right)_{p}, \quad \eta=\frac{1}{\rho_{g} c_{g}}-\varepsilon .
\end{gathered}
$$

Here $\rho_{j}$ and $c_{j}(j=g, i, h)$ are the density and specific heat of $j$-th phase; $\rho_{s k}, \lambda_{s k}$ and $c_{s k}$ are density, thermal conductivity and specific heat of the porous medium skeleton; $v_{g}$ is the gas velocity; $\varepsilon$ and $\eta$ are the throttling and adiabatic coefficients; $L_{h}$ is the heat of hydrate decomposition; $\rho c$ and $\lambda$ are the specific volumetric heat capacity and the thermal conductivity coefficient of the system of "porous medium - saturating substance".

To calculate the gas compressibility factor $z$ we use the Latonov-Gurevich equation:

$$
z=\left(0,4 \cdot \lg \left(T / T_{c}\right)+0,73\right)^{p / p_{c}}+0,1 \cdot p / p_{c} .
$$

Temperature and pressure in the zone of the hydrate decomposition are interrelated by the phase equilibrium condition $[2,7]$ :

$$
T=T_{0}+T_{*} \ln \left(p / p_{s 0}\right),
$$

where $p_{s 0}$ is the equilibrium pressure corresponding to the initial reservoir temperature $T_{0}$; $T_{*}$ is the empirical parameter which depends on the type of gas hydrate and the phases to which it decomposes.

\section{Calculation results}

The constructed system of equations was solved numerically using the implicit difference scheme, the tridiagonal matrix algorithm, the simple iteration method, and the original author's method to take into account the phase transition "hydrate - gas and ice".

The calculations were carried out at the following parameters $[2,7,8]: p_{0}=2.8 \mathrm{MPa}$; $T_{0}=270.15 \mathrm{~K}\left(-3^{\circ} \mathrm{C}\right) ; \quad r_{w}=0.1 \mathrm{~m} ; \quad R_{k}=500 \mathrm{~m} ; \quad m=0.1 ; \quad k=10^{-14} \mathrm{~m}^{2} ; \quad S_{h 0}=0.2 ;$ $p_{c}=4.6 \mathrm{MPa} ; \quad T_{c}=190.56 \mathrm{~K} ; \quad T_{*}=30 \mathrm{~K} ; \quad p_{s 0}=2.54 \mathrm{MPa} ; \quad c_{s k}=1000 \mathrm{~J} /(\mathrm{kg} \cdot \mathrm{K}) ;$ $c_{h}=2080 \mathrm{~J} /(\mathrm{kg} \cdot \mathrm{K}) ; \quad c_{i}=2060 \mathrm{~J} /(\mathrm{kg} \cdot \mathrm{K}) ; \quad \lambda_{s k}=1.5 \mathrm{~W} /(\mathrm{m} \cdot \mathrm{K}) ; \quad \lambda_{h}=0.45 \mathrm{~W} /(\mathrm{m} \cdot \mathrm{K}) ;$ $\lambda_{i}=2.2 \mathrm{~W} /(\mathrm{m} \cdot \mathrm{K}) ; \quad R_{g}=518.3 \mathrm{~J} /(\mathrm{kg} \cdot \mathrm{K}) ; \quad \rho_{s k}=2000 \mathrm{~kg} / \mathrm{m}^{3} ; \quad \rho_{h}=900 \mathrm{~kg} / \mathrm{m}^{3} ;$ $\rho_{i}=900 \mathrm{~kg} / \mathrm{m}^{3} ; G=0,12 ; L_{h}=166 \mathrm{~kJ} / \mathrm{kg} ;$ the spatial coordinate step $\Delta r=0.01 \mathrm{~m}$ at $r \in\left[r_{w}, 10\right], \Delta r=0.1 \mathrm{~m}$ at $r \in(10,100]$ and $\Delta r=1 \mathrm{~m}$ at $r \in\left(100, R_{k}\right]$; calculation time $t_{\text {end }}=30$ days; time step $\Delta t=60 \mathrm{~s}$; the values $c_{g}, \lambda_{g}, \mu_{g}$ were determined by interpolation of table data for methane.

The testing of the software program was carried out by comparing the results of numerical experiments and calculations carried out taking into account self-similar solutions from [2]. This comparison showed a satisfactory agreement. 
Figure 2 shows that depending on the gas mass flow rate $Q$, three cases are possible. The first case is characterized by the absence of the hydrate decomposition (line 1), in the second case the dissociation of the gas hydrate completely occurs at the frontal boundary (line 2), and the third case is characterized by the extended volume zone of phase transitions (lines 3 and 4).
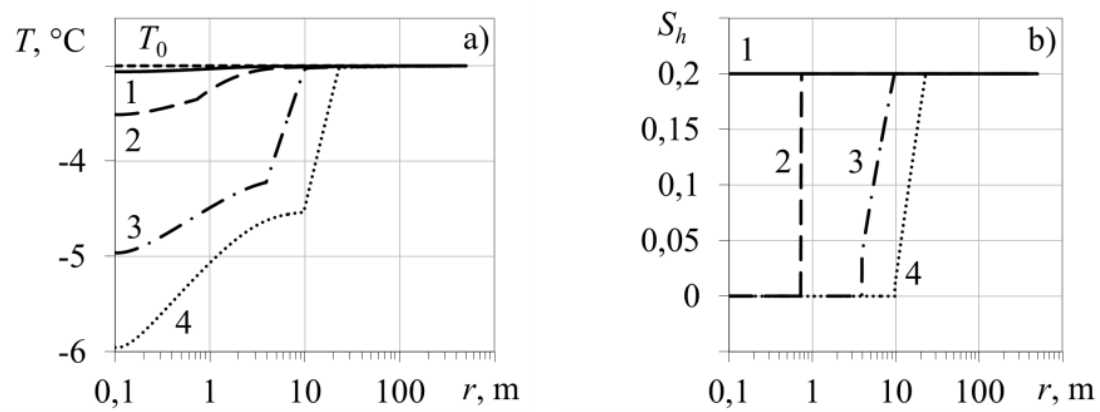

Fig. 2. The distributions of temperature (a) and hydrate saturation (b) by radial coordinate. Lines 1, 2, 3 and 4 correspond to the mass flow rate $Q=100,300,500$ and $700 \mathrm{~kg} /($ day $\cdot \mathrm{m})$.

Figure 2 shows that the reservoir temperatures is always either equal to or lower the initial reservoir temperature $T_{0}$, which is due to absorption of the latent heat of the phase transition and the decrease in gas temperature during filtration due to the adiabatic cooling and Joule-Thomson effects. Consequently, in the case of the negative initial reservoir temperature, the dissociation of gas hydrate will always produce gas and ice. In addition, at large values of the mass flow rate of gas extraction (large values of the pressure gradient) the volume zone of hydrate decomposition arises (lines 3 and 4), and with an increase in the value of $Q$ it becomes impossible to examine the process using the frontal scheme of hydrate decomposition. Also Fig. 2 shows that an increase in the mass flow rate of gas extraction leads to an increase in the extent of the volume zone of hydrate decomposition (second zone) and an increase in the fraction of the dissociated hydrate (lines 3 and 4).

This work was supported by the Grant of the President of the Russian Federation for State Support of Leading Scientific Schools of the Russian Federation (NSh-6987.2016.1).

\section{References}

1. Y.F. Makogon, S.A. Holditch, T.Y. Makogon, J. Petroleum Science and Eng. 5654 (2007)

2. M.K. Khasanov, N.G. Musakaev, I.K. Gimaltdinov, J. Eng. Phys. Thermophys. 88, 1052 (2015)

3. G.G. Tsypkin, Doklady Physics 46, 806 (2001)

4. V.I. Vasil'ev, V.V. Popov, G.G. Tsypkin, Fluid Dynamics 41, 599 (2006)

5. G.G. Tsypkin, J. Eng. Phys. Thermophys. 74, 1083 (2001)

6. V.Sh. Shagapov, N.G. Musakaev, M.K. Khasanov, Int. J. Heat Mass Transfer 84, 1030 (2015)

7. V.Sh. Shagapov, M.K. Khasanov, N.G. Musakaev, Ngoc Hai Duong, Int. J. Heat Mass Transfer 107, 347 (2017)

8. N.G. Musakaev, S.L. Borodin, Heat Mass Transfer 52, 981 (2016) 